\title{
Plasma, Salivary, and Urinary Oxytocin in Anorexia Nervosa: A Pilot Study
}

\author{
Elizabeth R. Hoffman, PhD ${ }^{a}$, Kimberly A. Brownley, $\mathbf{P h D}^{\mathrm{b}}$, Robert M. Hamer, $\mathrm{PhD}^{\mathrm{b}, \mathrm{c}}$, and \\ Cynthia M. Bulik, PhD ${ }^{a, b}$ \\ aDepartment of Nutrition, Gillings School of Global Public Health, University of North Carolina at \\ Chapel Hill, 2200 McGavran-Greenberg Hall, CB \#7461, Chapel Hill, NC 27599-7461, United \\ States, elizabeth_hoffman@med.unc.edu, cbulik@med.unc.edu \\ ${ }^{b}$ Department of Psychiatry, University of North Carolina at Chapel Hill, 101 Manning Drive, CB \\ \#7160, Chapel Hill, NC 27599-7160, United States, kim_brownley@med.unc.edu \\ 'Department of Biostatistics, Gillings School of Global Public Health, University of North Carolina \\ at Chapel Hill, 3101 McGavran-Greenberg Hall, CB \#7420, Chapel Hill, NC 27599-7420, United \\ States, hamer@unc.edu
}

\begin{abstract}
While oxytocin (OT) has the potential to be an informative biomarker of social functioning in patients with eating disorders, the burden of invasive blood draws or lumbar punctures limits OT study. Salivary and urinary OT measurements may be advantageous, as they require less invasive sampling techniques which could be conducted in a wider variety of settings. Yet, the degree to which the concentration of OT in these fluids is correlated with blood levels is uncertain, as is the impact of vomiting on salivary secretion of OT. Therefore, we compared contemporaneously sampled OT concentration in blood, saliva, and urine from twenty women acutely ill with anorexia nervosa. Salivary OT was positively correlated with plasma OT in patients with no history of selfinduced vomiting $(r=0.89)$, but correlation was lower in those with recent history of self-induced vomiting $(r=0.42)$. Urinary and plasma OT were not well-correlated $(r=0.13)$, suggesting preliminarily that collection of plasma OT remains the method of choice. Self-induced vomiting in eating disorders may limit the applicability of salivary sampling for OT.
\end{abstract}

\section{Keywords}

oxytocin; plasma; saliva; urine; eating disorder; self-induced vomiting

\footnotetext{
(C) 2012 Elsevier Ltd. All rights reserved.
}

Correspondence to: Elizabeth Hoffman, UNC Eating Disorders Program, University of North Carolina at Chapel Hill, CB \#7160, 101 Manning Drive, Chapel Hill, NC 27599-7160, United States, Voice: (919) 9663065 Fax: (919) 843-8802, elizabeth_hoffman@med.unc.edu.

Publisher's Disclaimer: This is a PDF file of an unedited manuscript that has been accepted for publication. As a service to our customers we are providing this early version of the manuscript. The manuscript will undergo copyediting, typesetting, and review of the resulting proof before it is published in its final citable form. Please note that during the production process errors may be discovered which could affect the content, and all legal disclaimers that apply to the journal pertain.

Contributors: All authors were involved in designing the research. Dr. Hoffman conducted the research and performed the statistical analysis with guidance from Dr. Hamer. Dr. Hoffman drafted the manuscript, and critical and intellectual feedback was provided by all other authors. All authors granted final approval of the version of the manuscript to be submitted.

Conflict of Interest: All other authors declare that they have no conflicts of interest. 


\section{Introduction}

Oxytocin (OT) is a hypothalamic neuropeptide associated with bonding and attachment behavior (Bello, White-Traut, Schwertz, Pournajafi-Nazarloo, \& Carter, 2008; Carter et al., 2007; Feldman, Weller, Zagoory-Sharon, \& Levine, 2007; Gouin et al., 2010; Light, Grewen, \& Amico, 2005), decreased anxiety and aggression (Mantella, Vollmer, Li, \& Amico, 2003; McCarthy, McDonald, Brooks, \& Goldman, 1996; Winslow et al., 2000), and dampened neuroendocrine response to stress (Light et al., 2004; Mantella, Vollmer, Rinaman, Li, \& Amico, 2004; Neumann, Torner, \& Wigger, 2000; Windle et al., 2004). Few studies have evaluated OT as a biomarker of attachment behavior and social functioning in patients with eating disorders due to the burden of invasive blood draws or lumbar punctures. Less invasive sampling of saliva or urine for OT may be advantageous, yet the evidence supporting these approaches is mixed, showing moderate correlations between plasma and salivary OT concentrations (Carter, et al., 2007; Feldman, Gordon, \& ZagoorySharon, 2011; Grewen, Davenport, \& Light, 2010) and no relationship between plasma and urinary OT (Feldman, et al., 2011) in healthy individuals. Furthermore, it is unknown whether self-induced vomiting, a common symptom in eating disorders that can cause salivary gland swelling (Lo Russo et al., 2008) and decreased salivary pH (Aframian, Ofir, $\&$ Benoliel, 2010), alters salivary secretion or assay of OT. Therefore, our objective for this pilot study was twofold; in patients with anorexia nervosa (AN) with and without recent history of self-induced vomiting: 1) to examine the correlation of plasma OT with salivary OT and 2) to examine the correlation of plasma OT with urinary OT, a new non-invasive option for OT measurement.

\section{Method}

\subsection{Participants}

The study was approved by the Biomedical Institutional Review Board of the University of North Carolina at Chapel Hill (UNC) and all participants provided informed consent. Twenty-five women over the age of 18 with a current diagnosis of AN were recruited from the UNC Eating Disorders Program inpatient unit. All women were diagnosed with AN by an attending psychiatrist according to DSM-IV criteria. Participants were recruited on a rolling basis after they had undergone at least one week of inpatient treatment. Of twentyfive potential participants identified, five declined participation due to concerns about having their blood drawn $(n=4)$ or concern that participation in a research study would interfere with treatment $(n=1)$. No further demographic or clinical information was available for those individuals who declined participation. For the final sample of twenty participants, medical records were evaluated to determine recent history of self-induced vomiting (defined as any report of self-induced vomiting in the month prior to admission), age, duration of eating disorder, current psychotropic medication use, and additional DSM-IV Axis I diagnoses including mood disorders, anxiety disorders, and substance-related disorders.

\subsection{Protocol}

All participants had undergone at least one week of inpatient treatment before participation. Two hours after their morning meal, participants were taken to the UNC Clinical and Translational Research Center (CTRC), and a medlock was placed. After a 20-minute relaxation period, serial blood and salivary samples were collected over fifteen minutes to decrease the effect of any pulsatile variability in OT (Cyranowski et al., 2008). At minutes 0 , 5,10 , and 15 , blood was collected into prechilled $6 \mathrm{~mL}$ ethylenediaminetetraacetic acid tubes, and 3000 KIUs of aprotinin were added to each tube per assay instructions to slow breakdown of the OT peptide. Before collecting saliva, participants thoroughly rinsed their 
mouth and lips to remove food and cosmetic residue. Chewing gum or drinking water was restricted during saliva collection. Participants were instructed not to swallow for one minute, to expel all saliva through passive drool into a prechilled CryoTube for one minute, then to rest for 30 seconds. This two and half minute process was repeated three times to fill each $1.8 \mathrm{~mL}$ CryoTube, resulting in two seven and a half minute saliva collecting periods beginning at minutes 0 and 7.5. Blood samples were immediately centrifuged at $3000 \times \mathrm{g}$ for 10 minutes at $4{ }^{\circ} \mathrm{C}$ and plasma pipetted into tubes and frozen with salivary samples at $-80^{\circ} \mathrm{C}$ until assay. With assistance and monitoring from nursing staff, participants began a 24-hour urine collection the same morning of the CTRC visit, ending with collection of the first morning urine the following day. Urine samples from the 24-hour collection were pipetted into $1.8 \mathrm{~mL}$ CryoTubes and frozen at $-80^{\circ} \mathrm{C}$ until assay.

\subsection{Enzyme Immunoassay Method}

Plasma, saliva, and urine samples were assayed in duplicate for OT by enzyme immunoassay (EIA) methods using a commercially available kit (Assay Designs, Ann Arbor, MI), with the addition of an extraction step that is recommended by the manufacturer and has been described in detail previously (Grewen, et al., 2010). The extraction reduces matrix interference and, through evaporation, concentrates the sample 3.2 times. Extraction efficiency for OT using this method was $93 \%$. After correcting for concentration produced by extraction, the lower limit of sensitivity of the OT EIA was $2.0 \mathrm{pg} / \mathrm{ml}$. The intra- and inter-assay variations for the assay, determined using a set control sample with known OT concentration on each plate and in each run, were $4.8 \%$ and $8 \%$, respectively. Assay Designs reports cross-reactivity for similar neuropeptides found in mammalian sera at less than 0.001 .

Urinary creatinine concentrations were measured using the VITROS CREA slide method (Ortho-Clinical Diagnostics, Inc. Rochester, NY). Urinary OT is expressed as the OT to creatinine ratio.

\subsection{Statistical Analysis}

All statistical analyses were conducted using SAS Version 9.2, and correlation analyses conducted using PROC CORR (SAS/STATß Software: Version 9.2, 2008). Pearson correlations were calculated to examine the linear association of salivary and urinary OT concentrations with plasma concentrations. Mean salivary OT (mean of two samples) and mean plasma OT (mean of four samples) for each individual were used in analyses. To investigate our hypothesis that self-induced vomiting might influence salivary OT concentrations, we calculated plasma-salivary correlations separately for individuals with and without recent history of self-induced vomiting. Due to the small sample size of this pilot study, we could not meaningfully test the difference between the two correlations, but could provide a gross comparison of the preliminary correlations as a guide for future larger investigations of this issue. One participant was missing salivary data due to inability to produce sufficient saliva for collection, and two participants were missing urinary data due to incomplete 24-hour urine collections.

\section{Results}

The mean age of the participants was 27.9 years ( $\mathrm{SD}=9.3$ years) with a mean duration of illness of 9.4 years ( $\mathrm{SD}=5.9$ years). Sixty-five percent of the sample had a comorbid depressive disorder, $35 \%$ had an anxiety disorder, and 20\% had a substance use disorder. Sixty-five percent of the sample was taking psychotropic medication at the time of the study. There were no significant differences between individuals with $(n=14)$ and without $(n=6)$ a recent history of self-induced vomiting in mean age, mean duration of illness, or frequency 
of depressive disorders, anxiety disorders, substance use disorders, or psychotropic medication use.

As shown in Figure 1, plasma OT and salivary OT concentrations were positively correlated, with the magnitude of this relation being considerably greater among individuals without a recent history of self-induced vomiting $(r=0.89)$ compared to those with recent history of self-induced vomiting $(r=0.42)$. Due to the small sample size of this pilot study, we could not meaningfully test the difference between the two correlations, but could provide this gross comparison of the preliminary correlations. Plasma OT and urinary OT were not well correlated $(r=0.13)$ in the full sample (Figure 2A). Two individuals had urinary OT concentrations of 92.6 and $56.4 \mathrm{pg} / \mathrm{mg}$ creatinine (with creatinine values in normal range), while urinary OT for the rest of the sample ranged from 1.1-26.1 pg/mg creatinine. Exploratory analysis excluding these two extreme samples demonstrated a positive correlation between plasma and urinary OT ( $r=0.51$; Figure $2 \mathrm{~B})$.

\section{Discussion}

The present findings, while preliminary, indicate that recent self-induced vomiting behavior may negatively impact the correlation of plasma OT with salivary OT, a problem for the use of this non-invasive method for measurement of OT in individuals with eating disorders marked by self-induced vomiting. Although this was a small sample pilot study that precluded hypothesis testing due to sample size and variability, these preliminary findings nonetheless raise concern and encourage further investigation of this observation. With reference to urinary OT, further study is needed to determine the normal range of urinary OT values expected. While this sample included two individuals with extreme urinary OT values, with a sample size of only 18 individuals for this new technique, it is unknown if these values represent true outliers or values in the higher range of a normal distribution. In this pilot sample, exclusion of these two extreme values yielded a larger positive correlation between plasma and urinary OT, supporting further larger studies of this non-invasive option for sampling OT.

Due to the limited scope of this pilot study, blood and saliva samples were collected at only one time point; in future studies it will be important to determine if self-induced vomiting behavior influences the correlation of plasma and salivary OT over time, limiting detection of changes in peripheral OT using saliva alone. Timing of sample collection represented a limitation in the analysis of urinary OT; while blood was collected over 15 minutes, urine was collected over 24 hours. Although a limitation, this was a deliberate aspect of the study design, as our objective at this early stage was to demonstrate proof of concept of collection and assay of OT in urine and gross correlation with plasma measures. Thus, timing may be at least partly responsible for the small positive correlation found between urine and plasma OT, but our results are similar to a recent study in a larger sample of healthy individuals that also found no significant relationship (Feldman, et al., 2011). Additional investigations into the utility of this sampling approach should determine the timing of urine sampling that most closely approximates plasma levels during experimental tasks (e.g., immediately after the task, thirty minutes later, one hour later, etc.) which may be complicated by interindividual variation in kidney function.

Although salivary measurement of OT is gaining favor (Carter, et al., 2007; Feldman, et al., 2011; Grewen, et al., 2010; White-Traut et al., 2009) due to less invasive sampling procedures, our findings warrant serious caution when considering salivary OT sampling in individuals with eating disorders marked by self-induced vomiting behaviors. Measurement of OT in urine may be another non-invasive option, but an initial challenge will be determination of appropriate timing of urine collection to produce meaningful results. 


\section{HIGHLIGHTS}

We examine correlations of plasma oxytocin with salivary and urinary oxytocin. We compare women with anorexia nervosa with and without self-induced vomiting. Plasmasalivary oxytocin correlation was lower in women with self-induced vomiting. Urinary oxytocin was not well correlated with plasma oxytocin.

\section{Acknowledgments}

Role of Funding Sources: This research was supported by grant UL1RR025747 from the National Center of Research Resources, National Institutes of Health. This funding source had no role in the study design, collection, analysis or interpretation of the data, writing the manuscript, or the decision to submit the paper for publication.

The authors would like to acknowledge Dr. Karen Grewen for her guidance with the salivary collection procedures.

\section{Abbreviations}

CTRC Clinical and Translational Research Center

EIA enzyme immunoassay

OT oxytocin

UNC University of North Carolina

\section{References}

Aframian DJ, Ofir M, Benoliel R. Comparison of oral mucosal $\mathrm{pH}$ values in bulimia nervosa, GERD, BMS patients and healthy population. Oral Diseases. 2010; 16(8):807-811. [PubMed: 20646236]

Bello D, White-Traut R, Schwertz D, Pournajafi-Nazarloo H, Carter CS. An exploratory study of neurohormonal responses of healthy men to massage. Journal of Alternative and Complementary Medicine. 2008; 14(4):387-394.

Carter CS, Pournajafi-Nazarloo H, Kramer KM, Ziegler TE, White-Traut R, Bello D, Schwertz D. Oxytocin: behavioral associations and potential as a salivary biomarker. Annals of the New York Academy of Sciences. 2007; 1098:312-322. [PubMed: 17435137]

Cyranowski JM, Hofkens TL, Frank E, Seltman H, Cai HM, Amico JA. Evidence of dysregulated peripheral oxytocin release among depressed women. Psychosomatic Medicine. 2008; 70(9):967975. [PubMed: 19005082]

Feldman R, Gordon I, Zagoory-Sharon O. Maternal and paternal plasma, salivary, and urinary oxytocin and parent-infant synchrony: considering stress and affiliation components of human bonding. Developmental Science. 2011; 14(4):752-761. [PubMed: 21676095]

Feldman R, Weller A, Zagoory-Sharon O, Levine A. Evidence for a neuroendocrinological foundation of human affiliation: plasma oxytocin levels across pregnancy and the postpartum period predict mother-infant bonding. Psychological Science. 2007; 18(11):965-970. [PubMed: 17958710]

Gouin JP, Carter CS, Pournajafi-Nazarloo H, Glaser R, Malarkey WB, Loving TJ, Kiecolt-Glaser JK. Marital behavior, oxytocin, vasopressin, and wound healing. Psychoneuroendocrinology. 2010; 35(7):1082-1090. [PubMed: 20144509]

Grewen KM, Davenport RE, Light KC. An investigation of plasma and salivary oxytocin responses in breast- and formula-feeding mothers of infants. Psychophysiology. 2010; 47(4):625-632. [PubMed: 20102537]

Light KC, Grewen KM, Amico JA. More frequent partner hugs and higher oxytocin levels are linked to lower blood pressure and heart rate in premenopausal women. Biological Psychology. 2005; 69(1):5-21. [PubMed: 15740822] 
Light KC, Grewen KM, Amico JA, Boccia M, Brownley KA, Johns JM. Deficits in plasma oxytocin responses and increased negative affect, stress, and blood pressure in mothers with cocaine exposure during pregnancy. Addictive Behaviors. 2004; 29(8):1541-1564. [PubMed: 15451123]

Lo Russo L, Campisi G, Di Fede O, Di Liberto C, Panzarella V, Lo Muzio L. Oral manifestations of eating disorders: a critical review. Oral Diseases. 2008; 14(6):479-484. [PubMed: 18826377]

Mantella RC, Vollmer RR, Li X, Amico JA. Female oxytocin-deficient mice display enhanced anxiety-related behavior. Endocrinology. 2003; 144(6):2291-2296. [PubMed: 12746288]

Mantella RC, Vollmer RR, Rinaman L, Li X, Amico JA. Enhanced corticosterone concentrations and attenuated Fos expression in the medial amygdala of female oxytocin knockout mice exposed to psychogenic stress. American Journal of Physiology- Regulatory, Integrative, and Comparative Physiology. 2004; 287(6):R1494-R1504.

McCarthy MM, McDonald CH, Brooks PJ, Goldman D. An anxiolytic action of oxytocin is enhanced by estrogen in the mouse. Physiology and Behavior. 1996; 60(5):1209-1215. [PubMed: 8916173]

Neumann ID, Torner L, Wigger A. Brain oxytocin: differential inhibition of neuroendocrine stress responses and anxiety-related behaviour in virgin, pregnant and lactating rats. Neuroscience. 2000; 95(2):567-575. [PubMed: 10658637]

SAS/STAT®. Software: Version 9.2. Cary, NC: SAS Institute Inc.; 2008.

White-Traut R, Watanabe K, Pournajafi-Nazarloo H, Schwertz D, Bell A, Carter CS. Detection of salivary oxytocin levels in lactating women. Developmental Psychobiology. 2009; 51(4):367-373. [PubMed: 19365797]

Windle RJ, Kershaw YM, Shanks N, Wood SA, Lightman SL, Ingram CD. Oxytocin attenuates stressinduced c-fos mRNA expression in specific forebrain regions associated with modulation of hypothalamo-pituitary-adrenal activity. Journal of Neuroscience. 2004; 24(12):2974-2982. [PubMed: 15044536]

Winslow JT, Hearn EF, Ferguson J, Young LJ, Matzuk MM, Insel TR. Infant vocalization, adult aggression, and fear behavior of an oxytocin null mutant mouse. Hormones and Behavior. 2000; 37(2):145-155. [PubMed: 10753584] 


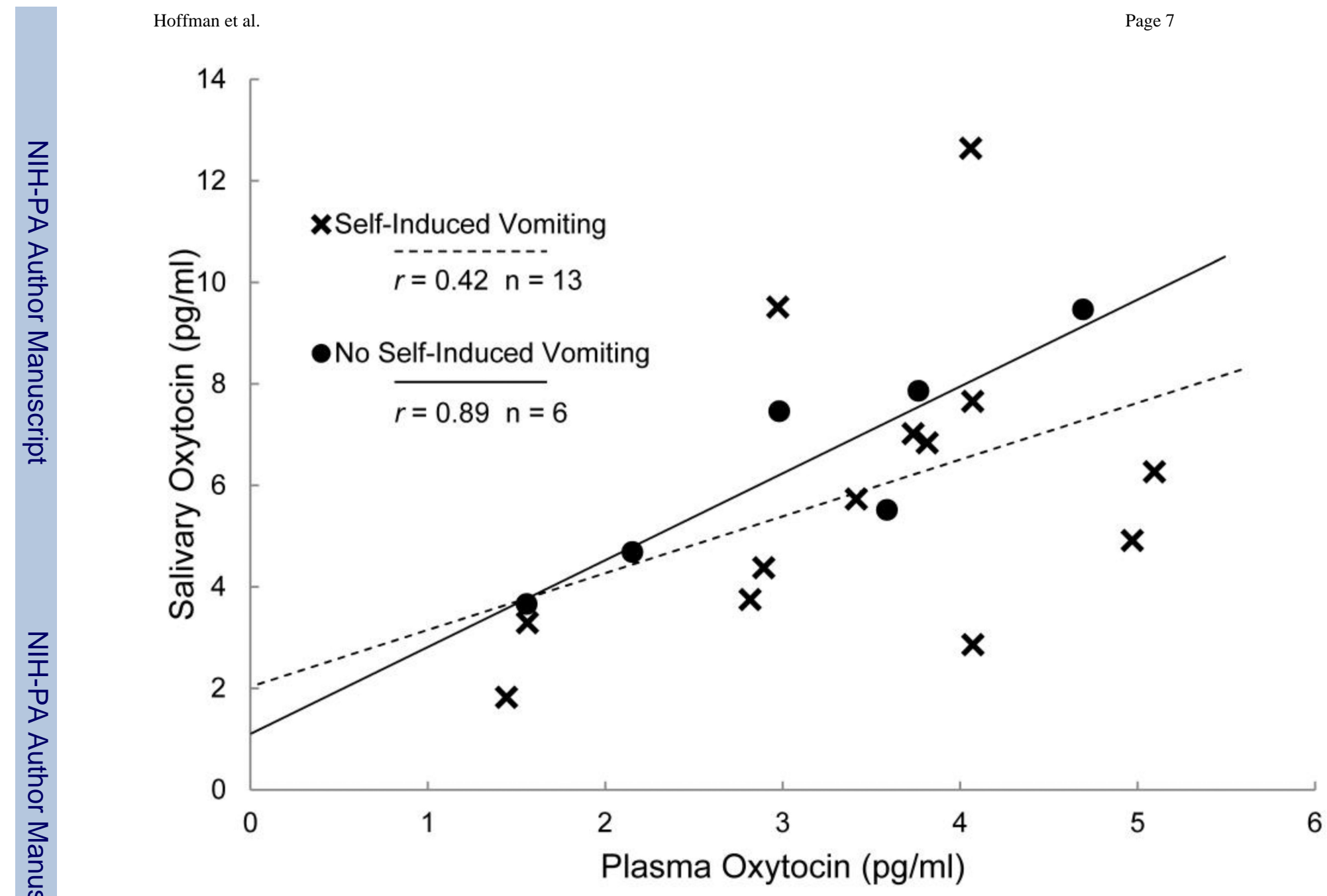

Figure 1.

Mean plasma oxytocin concentrations plotted against mean salivary oxytocin concentrations for individuals with and without recent history of self-induced vomiting. Pearson correlations are presented for each group. 

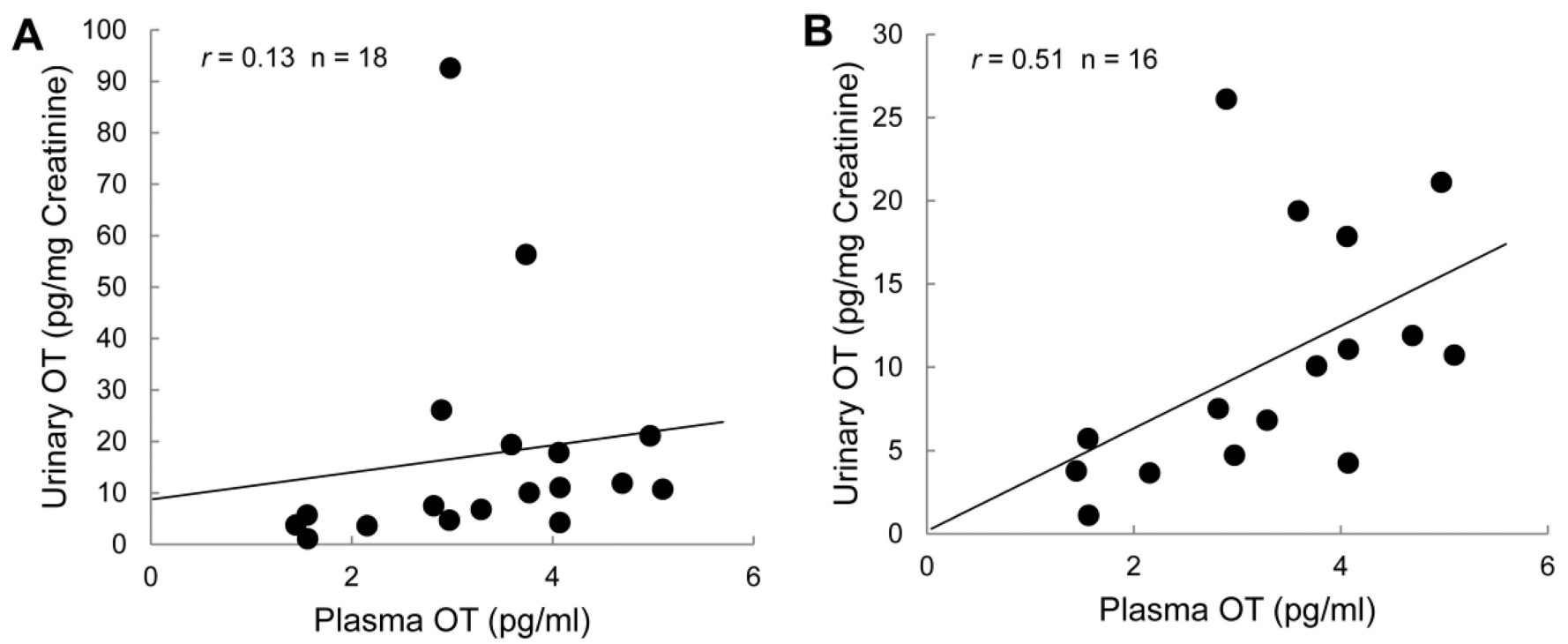

Figure 2.

Mean plasma oxytocin concentrations plotted against urinary oxytocin concentration for (A) the entire sample and (B) the sample excluding two individuals with normal creatinine but extreme values for urinary oxytocin (92.6 and $56.4 \mathrm{pg} / \mathrm{mg}$ creatinine). Pearson correlations are presented. 